\section{High resolution CT of the lungs - the Groote Schuur experience}

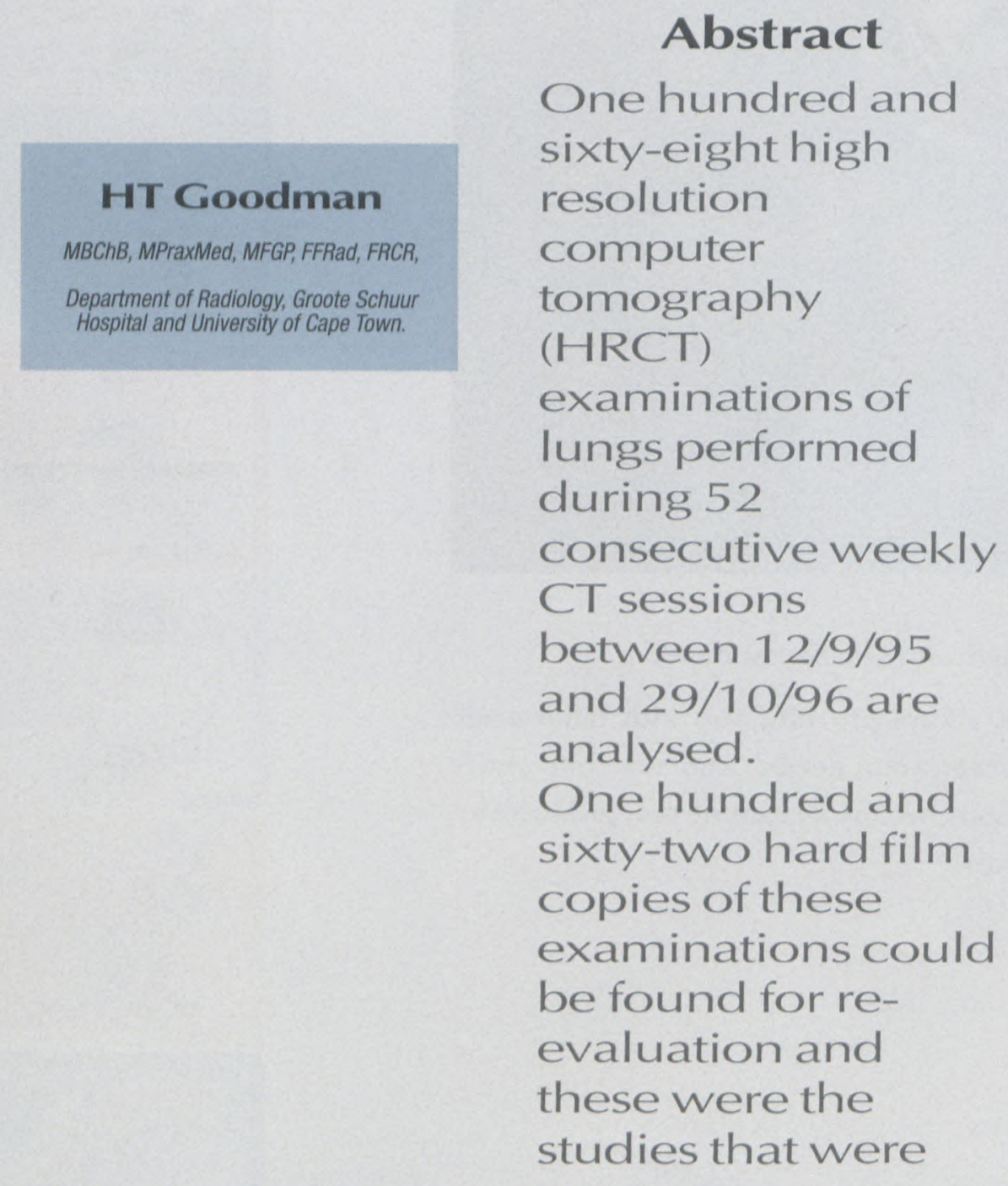

analysed. They form the bulk of the HRCT examinations of the lungs done at our institution during this period.

At our institution high resolution of the lungs is done to answer the following questions:

1. What was the extent of lung involvement of a given condition?

2. Was there a recognisable cause to account for an haemoptysis; and less often to account for pulmonary hypertension?

3. Was any lung parenchymal process recognisable?

4. Was the HRCT picture supportive of a suggested clinical condition?

5. What condition did the HRCT examination suggest to account for the radiological or lung function change or for the patients' symptoms?

The author will attempt to describe his ability to answer the above questions. He must stress that pathological proof for the accepted diagnosis of each case is not always present. In those patients in whom there is no pathological diagnosis, the assessment of his ability to answer the question posed is based on the clinical diagnosis. This clinical assessment has been obtained from a close working relationship with the referring practitioners; from discussions at regular clinico-radiological and monthly clinico-radiological-pathological meetings at which many of the HRCT examinations were reviewed; as well as from a review of the clinical and histological records of 59 patients. 
from page 10

\section{What was the extent of lung involvement?}

The easiest requirement to fulfill was "Record the distribution and extent" of any specific condition. This involved 40 patients suffering from:

a) Mycobacterium infection (n14)

b) cryptogenic fibrosing alveolitis (CFA) also known as usual interstitial pneumonitis (UIP) (n14)

c) emphysema (n5)

d) bronchiectasis (n5), and

e) alveolar proteinosis (n2)

The patients with emphysema, often of a panlobular distribution, were usually those who were candidates for either lung reduction surgery or bullectomies.

The HRCT description of the distribution and extent of bronchiectatic (Figure 1) involvement and of

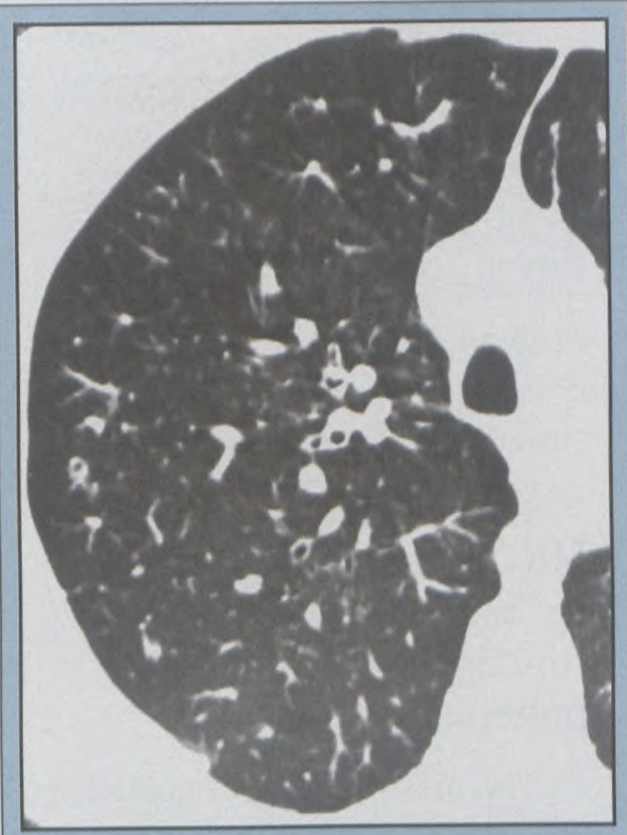

Figure 1: Early bronchiectatic change. On a section at the level of the aortic arch one can see a thick walled dilated bronchus peripherally. Bronchi are not normally seen in the peripheral portion of the lung.

Mycobacterium infection (Figure 2) was usually accepted as valid. This was often without surgical or other confirmation.

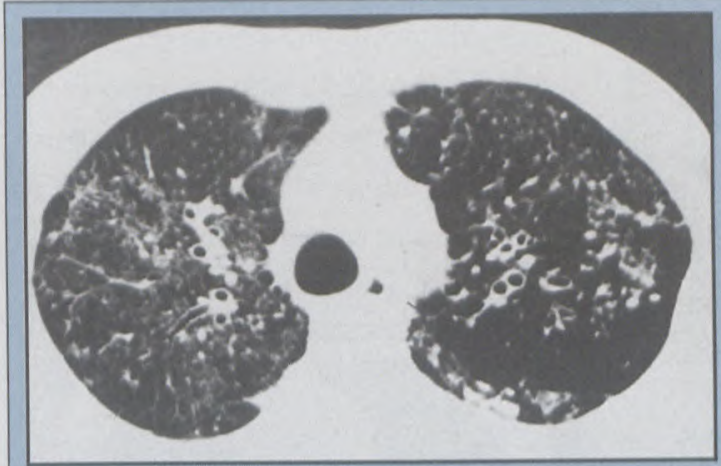

Figure 2: Mycobacterium tuberculosis organisms were cultured from the patient's sputum. On the HRCT scan diffuse bilateral bronchiectasis can be seen. Irregular opacification is present with distortion of lung anatomy and emphysema. Most of the changes were in the upper lung zones.

The displacement, distortion and obscuration of landmarks such as the fissures by the disease process made it difficult to localise the radiological change accurately to a specific lobe or segment of a lobe on some of the scans. This difficulty occurred mainly in mycobacterial infection and in bronchiectasis. The author is not aware of surgical findings significantly different from the HRCT interpretation in this group.

We found a composite diagram produced by The Health Sciences Division, Eastman Kodak Company, Rochester, New York; and based on an article by NRL Bechai and DJWise in an in-house technical publication, most helpful for localising lesions to specific lung segments on cross sectional examinations.

Our institution participates in an international assessment of the response of patients with cryptogenic fibrosing alveolitis (CFA) to various treatment regimens; and HRCT examination plays a part in this evaluation.

When reassessing the hard copies of the HRCT examination without knowing what he had reported at the time of the investigation, the author did at times give a different opinion from the one that he had previously expressed as to whether the extent of the disease process had changed since an earlier examination. This, in his opinion, emphasises the difficulty which can be experienced in making these assessments.

The author suggests that the image on the recording film be as large as is practical. This makes for easier subsequent interpretation from the hard film. The pictures should be recorded at the same Hounsfield centre and window settings on each occasion, and at the same camera settings, if comparisons between different examinations are to be made. Specific sections, at the same anatomical level, should be compared.

\section{Was there a recognisable cause to account for an haemoptysis or for pulmo- nary hypertension?}

The examinations of the $13 \mathrm{pa}-$ tients who presented with what was usually a significant haemoptysis were relatively easy to interpret. The clinical question was nearly always: Was there evidence of a condition such as bronchiectasis - or of a bronchial lesion - to account for the haemoptysis; and if present what was its distribution? In four patients bronchiectasis was delineated. No other significant lesion was recognised.

Most of the examinations were done with $2 \mathrm{~mm}$ thick scans at $10 \mathrm{~mm}$ intervals. Webb et al ${ }^{1}$ suggest that the subcarinal area to the level of the pulmonary veins should be examined with 4 or $5 \mathrm{~mm}$ thick contiguous sections when searching for a cause of haemoptysis. 


\section{from page 11}

Four patients also presented with pulmonary hypertension with no clinically recognisable cause. In two of the four patients obliterative or constrictive bronchiolitis (Figure 3) was thought to be present, and in one patient emphysema was demonstrated.

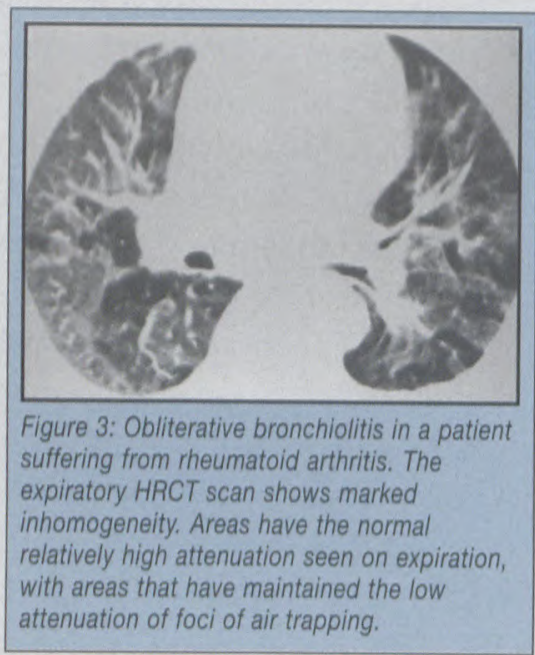

On the three occasions on which the cause of radiologically recognised hilar adenopathy was sought, no recognised pulmonary abnormality was seen on the HRCT examination.

\section{Was any lung parenchymal process recognisable?}

In 28 patients the main question posed was: "Was there evidence of diffuse pulmonary disease?" The clinical conditions which prompted this question included pleural disease which obscured the underlying lungs, evidence of extrapulmonary sarcoidosis or tuberculosis, a diagnosis of a collagen vascular disease, and the possibility of micronodular metastases or of bronchiectasis. A diagnosis of emphysema, CFA, early bronchiectasis, tuberculosis and of possible previous embolic disease was suggested on occasions; but the vast majority of these examinations were thought to show no diffuse pulmonary process.
In this group one known error was made. In a patient who had received a single lung transplantation and who was feeling generally unwell, a micronodular pattern was recognised on the HRCT examination (Figure 4),

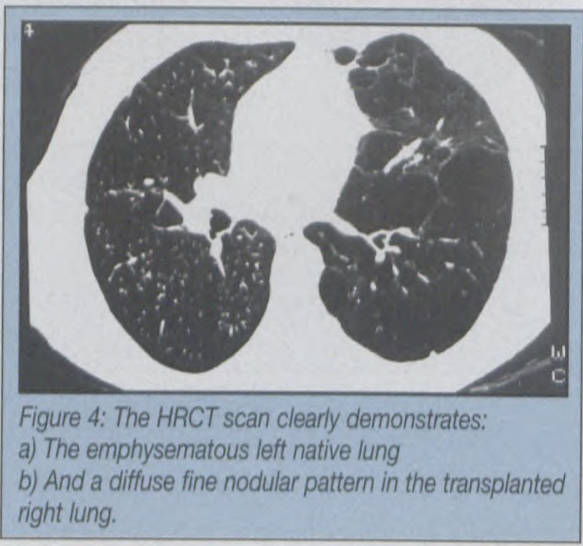

but its significance was not adequately emphasised. The patient deteriorated two weeks later and evidence of diffuse alveolar damage and of focal acute bronchiolitis was seen in the lung specimen. No causative organism was found.

\section{Was the HRCT picture supportive of a suggested clinical condition?}

On 30 occasions the radiologist was asked to confirm the presence of

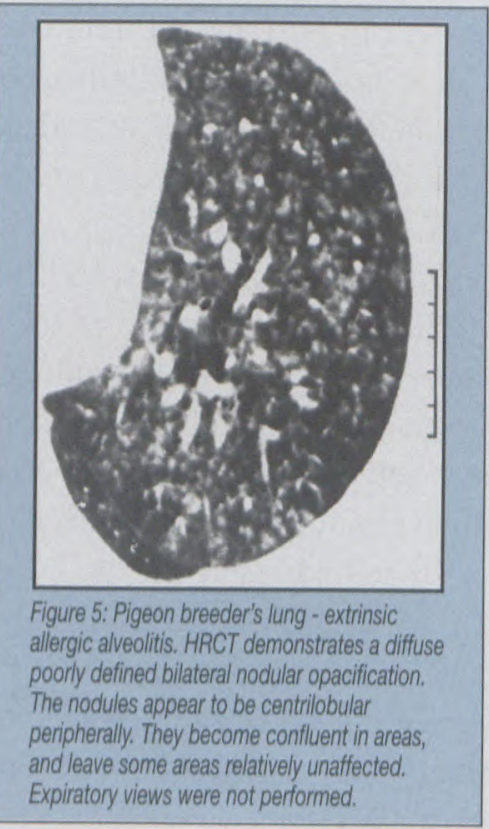

a specific condition suspected clinically or on a plain chest radiograph. These conditions included CFA, extrinsic allergic alveolitis (EAA) (Figure 5), lymphagenitic carcinomatosis, bronchiectasis and emphysema. On the vast majority of the examinations that have been included under this heading, the HRCT picture was thought to be suggestive of, or in keeping with, the proposed diagnosis. On occasions an extra feature was found. These included a mass lesion in a patient with CFA (Figure 6), a mycetoma in a tuberculous

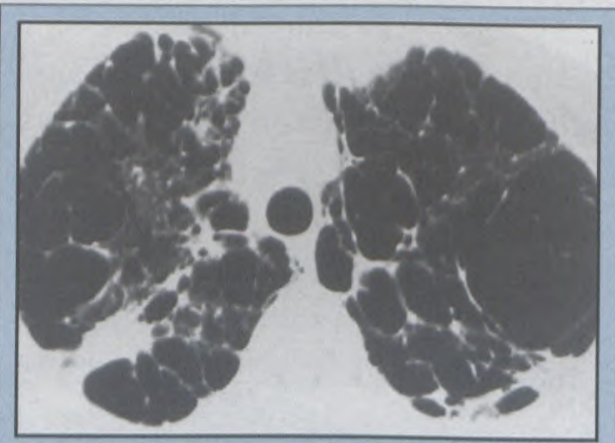

Figure 6: Cryptogenic fibrosing alveolitis, emphysema and carcinoma of the lung. The significance of the area of opacification in the right lung was missed. This was a carcinoma in a patient with both honeycomb changes of CFA which were better seen on the lower zones and bullous emphysema.

cavity and, more often, a combination of emphysema and CFA or of bronchiectasis and emphysema.

\section{What condition did HRCT suggest to account for radiological or lung function change or for patients' symptoms?}

The most difficult examinations were those examinations on patients in whom the clinical diagnosis was not clear and the $\mathrm{CT}$ was requested in an effort to elucidate the problem.

There were 47 such examinations. The questions posed varied considerably, and it is difficult to group the questions into meaningful smaller 


\section{High resolution CT of the lungs - the \\ Groote Schuur experience}

\section{from page 12}

divisions. Often the clinical differential diagnosis would include CFA and sarcoidosis. The possibility of lymphangitic carcinomatosis, alveolar proteinosis (Figure 7), bronchiectasis,

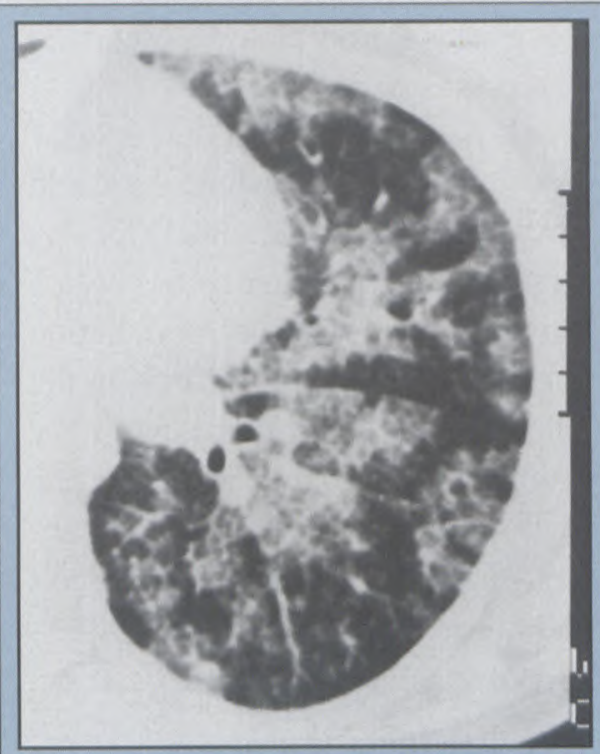

Figure 7: Alveolar proteinosis. A widespread patchy bilateral, but asymmetrical (not shown) ground glass opacification is present. It has areas with well defined edges. Thickened septal lines can be seen crossing the areas of opacification.

cryptogenic fibrosing alveolitis, histiocytosis X (Langerhans cell histiocytosis), extrinsic allergic alveolitis, drug reaction, emphysema and others were all raised for consideration in the differential diagnoses.

Neither the differential diagnosis of specific changes seen on HRCT examinations nor the classical changes seen in specific disease processes will be discussed as both of these aspects are extremely well covered in many original articles and in the two magnificent monographs by Webb, Muller and Naidich ${ }^{1}$ and by Stern and Swensen ${ }^{2}$. The author does however wish to highlight some of his difficulties in interpreting HRCT lung examinations and to refer to some of the points he has learned. These include:

- The non-uniform nature of lymphangitic carcinomatosis involvement of the lungs. This point is emphasised by others; but the author found that he needed to become accustomed to it in practice. The HRCT picture of this condition also varied considerably.

- Similarly, sarcoidosis of the lungs often caused a non-uniform alteration; and the nodular change appeared to involve some of the bronchovascular bundles and to spare others. Once again the HRCT of the condition showed considerable variation (Figure 8 )

- The author also had to learn the very

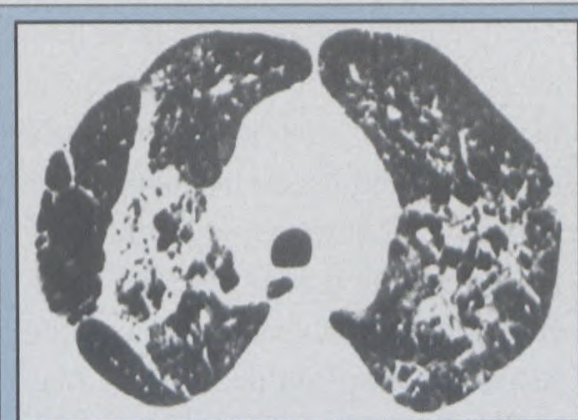

Figure 8: Sarcoidosis. Bilateral, central, irregular opacification with distortion of lung morphology is present. Associated traction bronchiectasis is seen Separate small nodules and emphysema are noted. The changes were predominantly in the medial and upper zones. Hilar and mediastinal lymphadenopathy was present (not shown). The histology reveals peribronchiovascular granulomata.

variable picture caused by cryptogenic fibrosing alveolitis, and also the lack of symmetry on occasions; and the fact that the bases did not always show the most extensive change (Figures 9 and 10). If the peripheral honeycomb change is distributed anteriorly in the upper zones, laterally in the mid zones and posteriorly in the lower zones, this supports a diagnosis of CFA.

- Early bronchiectasis continues to be a difficult diagnosis at times. Unfortunately the use of a very slow scanner, 4 seconds, with a minimum slice thickness of $2 \mathrm{~mm}$, compounds the difficulty. The author is impressed by the fact that there does not always appear to be loss of volume of the surrounding lung in regions of bronchiectasis.
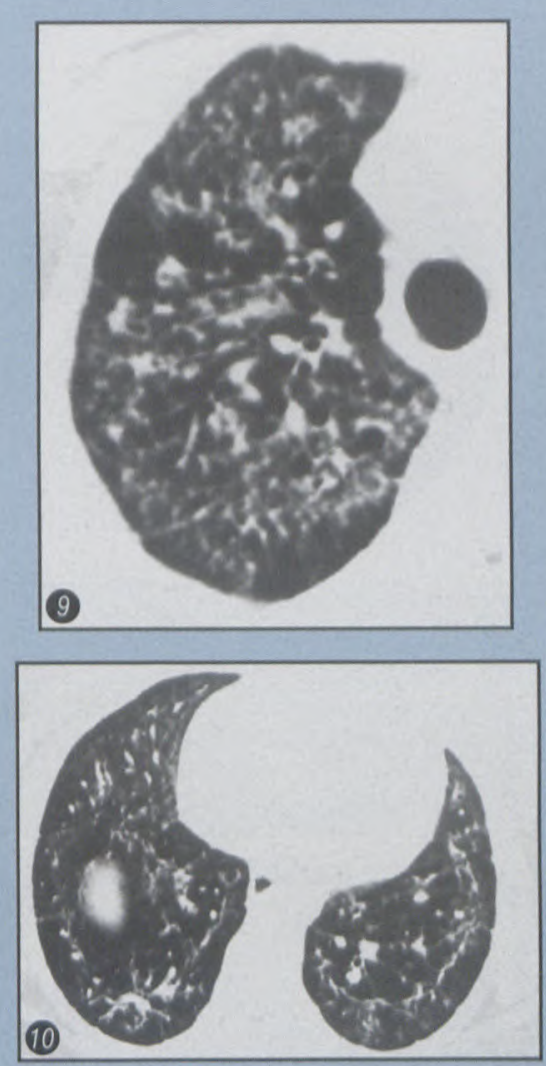

Figure 9 and 10: Cryptogenic fibrosing alveolitis. The diagnosis is supported by an open lung biopsy. Although the carinal level did show a peripheral distribution of the fibrotic changes, the two sections demonstrate an unusual distribution of irregular opacification and distortion of the normal lung morphology. In patients with CFA the fibrosis is usually more peripheral and with a greater lower zone predominance.

- The narrowing down of the relevant differential diagnosis of a micronodular pattern continues to cause the author difficulties (Figures 11,12 and 13).

- Bronchiectasis and involvement of tuberculotic disease of the lung may cause displacement of the fissures. This sometimes makes the placement of the condition into specific lung segments difficult.

- The author found it difficult at times to suggest whether the changes caused by tuberculosis were due to any active process or not (Figure 2). He suspects that he did not pay enough attention to the "tree in bud" 


\section{High resolution CT of the lungs - the \\ Groote-Schuur experience}

\section{from page 13}

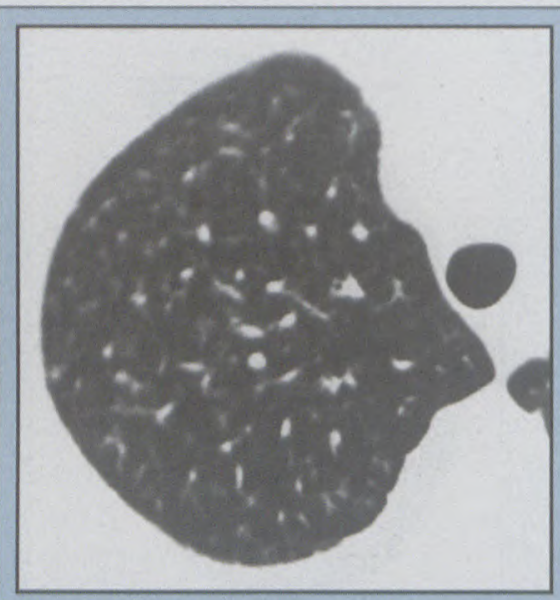

Figure 11: HRCT of a 42 year old woman patient with smoker's bronchiolitis. A diffuse, predominantly upper zone bilateral fine, poorly defined nodular pattern is present. An expiratory examination was not done. Histology revealed peri-bronchiolar interstitial infiltrate and smoker's pigment.

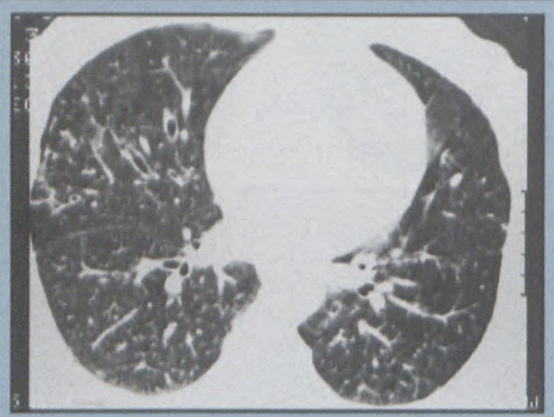

Figure 12: Hut lung. The HRCT demonstrates: a) A fine nodular pattern; b) Bronchiectatic change: and c) Inhomogeneity of attenuation.

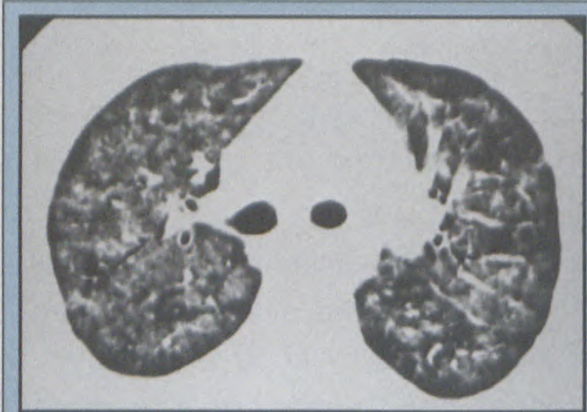

Figure 13: Interstitial pneumonia, probably viral in nature, in a 13 year old boy. A bilateral, diffuse ground glass opacification with a nodular component is present. The upper zones were more intensely affected than the lower lungs.

appearance stressed by Webb et al. Stern and Swensen ${ }^{2}$ state that cavitation in reactivation tuberculosis seen on HRCT does not in itself indicate disease activity.
- In his experience with those radiologists learning to do HRCT examinations the author found that motion artefacts causing the "star" artefact, and dependent hyperattenuation, caused the most easily avoidable errors in interpretation. He found that examinations done to confirm or negate the presence of asbestosis did not always include examinations in the prone position.

In a patient with a classical clinical presentation and supportive investigatory findings of a specific diffuse lung condition, the support of a typical HRCT examination picture may obviate the need for an histological examination. If however, any of the findings, including that of the HRCT examination, is not characteristic of the suspected disease, a lung biopsy may still be necessary (Figure 14)

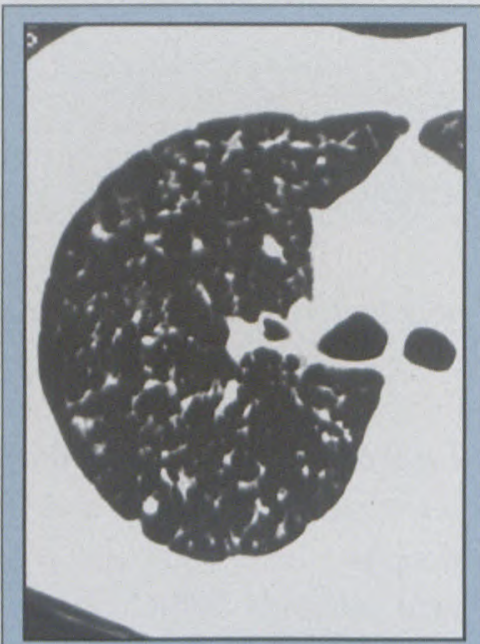

Figure 14: The HRCT scan of a 34 year old woman patient. On this examination the difficulty that can be experienced in interpreting a diffuse cystic change is demonstrated. The histology did not confirm a diagnosis of histiocytosis $X$ or of lymphangio-myomatosis. Clinically the diagnosis is thought to be histiocytosis $X$ or emphysema. Diffuse bilateral symmetrical cystic spaces are present. Although no very distinct walls are seen, these are nevertheless thought to be present. Most of the lung is involved. There was increased attenuation of mainly the posterior lung between the cysts on expiration (not shown).
In conclusion the author has enjoyed learning about, and doing HRCT examinations of the lung. He thinks that the relatively old machine that he uses and his film format detract from the clarity of the pictures especially as reproduced on hard film.

He has sorely missed the presence of a colleague with whom he could discuss perplexing studies. Working closely with the clinicians has been a particular source of joy, stimulation and instruction. Unfortunately in the subsection of the patients with the more difficult clinical problems the high resolution CT examination appears to have been the most difficult to interpret, and to have the highest proportion of errors of interpretation.

\section{Acknowledgement}

Thanks to Ms B Neumann for her secretarial help.

\section{References}

1. Webb WR, Müller NL and Naidich DP. High resolution $\mathrm{CT}$ of the lung. Second edition. Philadelphia: Lippincott-Raven Publishers 1996.

2. Stern EJ and Swensen SJ. High-resolution CT of the chest. Comprehensive atlas. Philadelphia: Lippincott-Raven Publishers. 1996. 